\title{
Disseminated mucormycosis presenting with acute renal failure
}

\author{
K.L. Gupta, ${ }^{1}$ Kusum Joshi, ${ }^{2}$ Brian J.G. Pereira ${ }^{1}$ and Kartar Singh ${ }^{3}$ \\ Departments of ${ }^{1}$ Nephrology, ${ }^{2}$ Pathology and ${ }^{3}$ Gastroenterology, Postgraduate Institute of Medical Education and \\ Research, Chandigarh-160012, India.
}

\begin{abstract}
Summary: An unusual presentation of disseminated mucormycosis as acute renal failure in a patient without any predisposing condition, is reported. The diagnosis was established at autopsy.
\end{abstract}

\section{Introduction}

Mucormycosis is an invasive fungal infection caused by members of the family Mucoraceae (genera Mucor, Rhizopus and Absidia). It was first elaborated as a clinical entity by Gregory et al. ${ }^{1}$ in 1943 , in association with diabetes mellitus. Subsequently its occurrence in patients with diabetic ketoacidosis, leukaemia, lymphomas, multiple myeloma, uraemia, burns, renal transplant recipients and those on corticosteroid and immunosuppressive therapy has been described.$^{2-4} \mathrm{We}$ report the clinicopathological features of a case of disseminated mucormycosis which presented with acute renal failure.

\section{Case report}

A 53 year old man presented with hiccoughs and vomiting for 15 days, 10-15 tarry stools with fresh blood for 3 days, oliguria followed by anuria for 3 days and altered sensorium for 2 days. He was a chronic smoker with a long history of cough with mucoid expectoration.

Physical examination revealed marked pallor and digital clubbing. Pulse was $120 / \mathrm{min}$, blood pressure $90 / 50 \mathrm{~mm} \mathrm{Hg}$ and temperature $37^{\circ} \mathrm{C}$.

Investigations showed haemoglobin of $3.41 \mathrm{mmol} / 1$, total leucocyte count $57.6 \times 10^{9} / 1$ with $74 \%$ neutrophils and $26 \%$ lymphocytes. Blood biochemistry showed sodium $110 \mathrm{mmol} / \mathrm{l}$, potassium $5.0 \mathrm{mmol} / \mathrm{l}$, calcium $1.88 \mathrm{mmol} / 1$, inorganic phosphorus $1.45 \mathrm{mmol} / 1$, urea $57.2 \mathrm{mmol} / \mathrm{l}$, creatinine $353.6 \mu \mathrm{mol} / \mathrm{l}$. Blood culture was sterile. Chest X-ray showed emphysematous lung fields with a patchy consolidation in the right upper zone. Ultrasonographic examination of the abdomen revealed bilaterally

Correspondence: K. Singh, M.D., D.M.

Accepted: 27 October 1986 enlarged kidneys with normal echo pattern and regular outlines. Upper gastrointestinal endoscopy revealed oesophagitis and 2 ulcers in the stomach besides several healed erosions.

Peritoneal dialysis was instituted twice to combat the uraemia. On the 10th day in hospital the patient developed fever and abdominal tenderness which progressed to peritonitis, septicaemia, shock and death.

At autopsy the lungs showed changes of anthracosis and emphysema with an abscess, $1.5 \mathrm{~cm}$ in diameter, in the right upper lobe. Microscopic examination of the abscess revealed a necrotic centre containing septate hyphae of Mucor with right angled branching pattern and invading the blood vessels in the adjoining lung tissue (Figure 1). Both kidneys had a variegated appearance showing large infarcts, ischaemic cortical necrosis, haemorrhages and exudates on the surface.

The artery and vein at the hilum, on both sides, showed approximately $90 \%$ occlusion of their lumina by thrombi. Microscopic examination of the kidney tissue revealed infarction and granulomatous inflammation with central necrosis, vasculitis and thrombosis of large and medium sized arteries and veins (Figure 2). Mucor hyphae were present in the interstitium, blood vessels, glomeruli and tubules. The hyphae were not seen in the giant cells, but were seen in the necrotic areas in the centre of the granulomas. The urinary bladder showed petechial haemorrhages over the mucosa, but no fungal hyphae. The pancreas was enlarged and adherent to the surrounding structures with areas of necrosis, and microscopic examination revealed Mucor hyphae. There were features of peritonitis with exudates over the kidneys, adrenals and mesentery containing the fungus. The intestines revealed ischaemic changes in the ileum and colon with vascular occlusion by thrombi containing fungal hyphae.

(C) The Fellowship of Postgraduate Medicine, 1987 


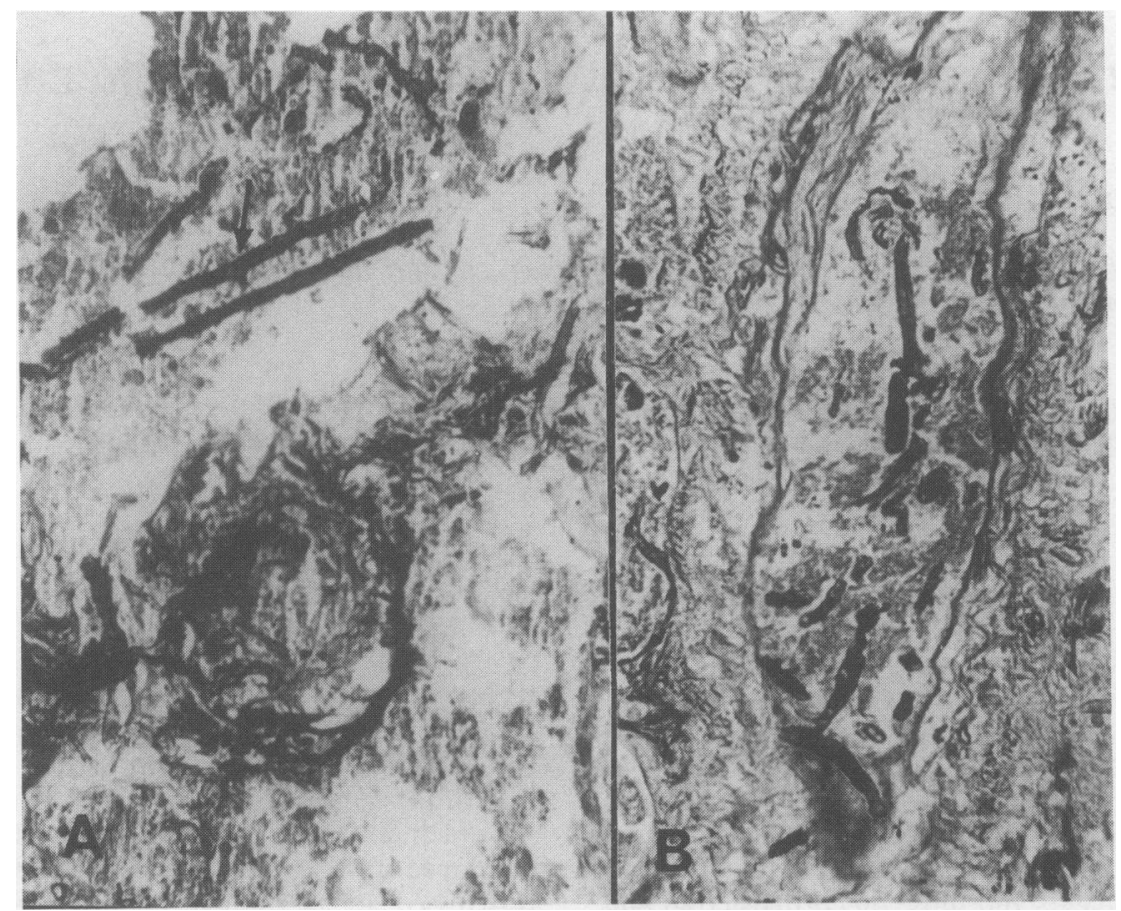

Figure 1 Photomicrographs from the lung showing: (A) mass of tangled fungal hyphae in the abscess cavity. A long hypha showing right angled branching is indicated (arrow); (B) vascular invasion by the fungi (Grocott stain $\times 600)$.

Thus this patient had disseminated mucormycosis with the primary focus most probably in the lung and secondary involvement of the kidneys, pancreas, intestines, mesentery and peritoneum.

\section{Discussion}

Fungi of the family Mucoraceae exist ubiquitously in the soil and are not usually pathogenic to human beings unless the host defences are compromised. Though diabetes mellitus, lymphomas, leukaemias and other immuno-suppressed states are the usual predisposing conditions, the disease is known to occur in apparently normal individuals. ${ }^{5}$ Our patient also did not have an obvious illness which could have facilitated the disseminated Mucor invasion.

The spectrum of mucormycosis includes its presentation as rhinocerebral, rhinoorbital, paranasal, cerebral, pulmonary, cutaneous, gastrointestinal, aural and disseminated disease. ${ }^{2}$ Disseminated mucormycosis as was present in our patient is invariably fatal and the diagnosis is seldom made during life due to the non-specificity of symptoms and signs. Parfrey, in a recent series, reported widely disseminated disease in $3 \overrightarrow{\vec{F}}$ out of the 33 cases of mucormycosis reviewed by him, ${ }^{2} \frac{}{3}$ whereas Meyer et al. had earlier found it in 10 out of $\bar{P}$ the 26 cases with underlying leukaemia of lymphoma. ${ }^{3}$

Pulmonary mucormycosis may present as an isolated involvement or as part of the disseminated 3 disease, wherein the lung is often the primary site of infection. Pulmonary vascular thrombosis and infarction have been present almost uniformly and it has $\delta$ been suggested that the cavities as seen in our patient represent liquefaction of pulmonary infarcts. ${ }^{5}$

Renal failure which was the predominant presenting feature in this patient is not common in mucormycosis, although renal involvement in the dissemin- $\bar{N}$

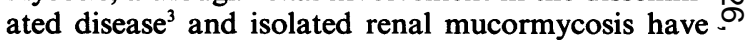
both been reported. ${ }^{7,8}$ The characteristic lesions of $N$ Mucor invasion involving the hilar as well as smaller $\underset{\mathrm{W}}{\mathrm{N}}$ vessels in both the kidneys with associated thrombosis, 음 infarction, ischaemic cortical necrosis and gran-o ulomatous inflammation seen in this case were respon- $\frac{\tau}{\Phi}$ sible for acute renal failure. The inappropriately low $\stackrel{\mathcal{D}}{\rightarrow}$ haemoglobin level was secondary to the complicating $\square$ gastrointestinal haemorrhage, which by itself was not $\stackrel{\vec{O}}{\vec{P}}$ the primary cause of renal failure. Gastrointestinal 


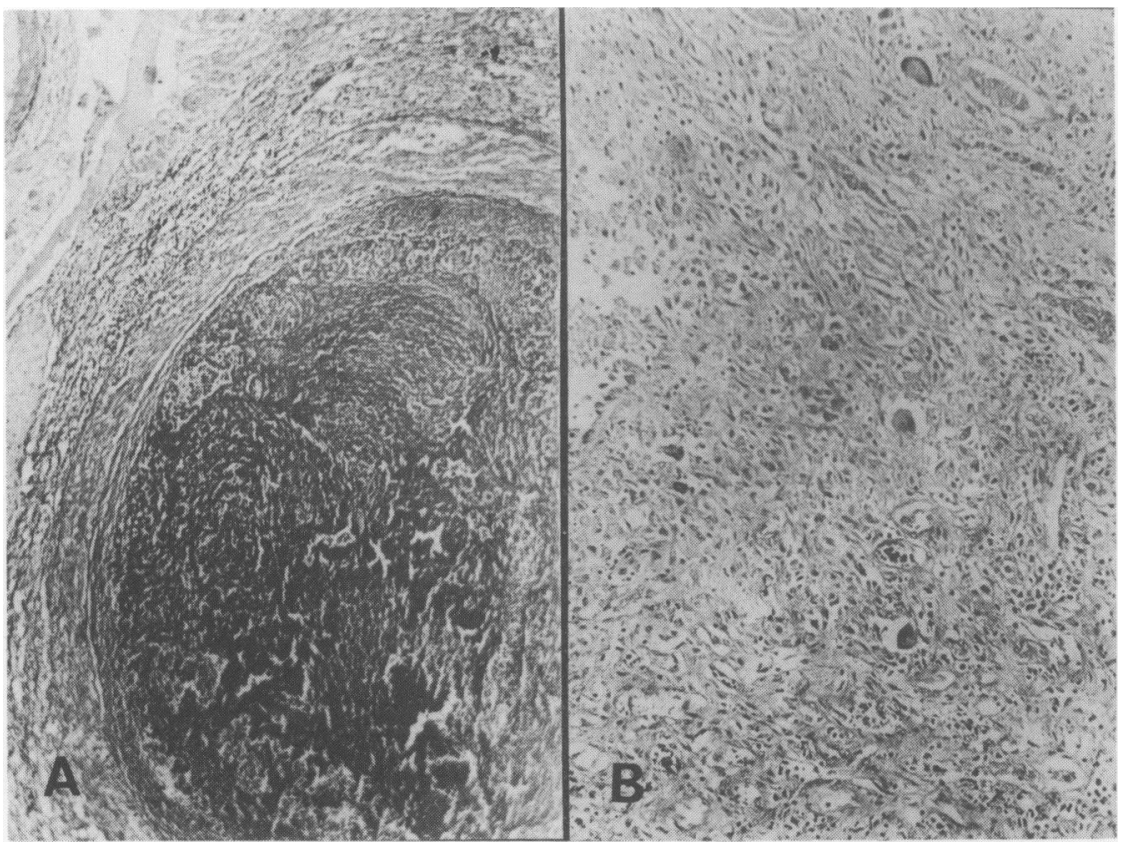

Figure 2 Photomicrographs of the kidney showing: (A) thrombotic occlusion and vasculitis of the hilar vessel (H \& $\mathrm{E} \times 60$ ); (B) granulomatous inflammation consisting of epithelioid cells, giant cells and a few lymphocytes with Mucor hyphae in an extracellular location (H \& $\mathrm{E} \times 150)$.

mucormycosis is rare and usually involves the stomach, with the colon next in frequency.,10 Involvement of the ileum, colon, pancreas and peritoneum as seen in this patient are also unusual features.

The diagnosis of mucormycosis should be made on histological grounds, as positive cultures may merely

\section{References}

1. Gregory, J.E., Golden, A. \& Haymaker, W. Mucormycosis of the central nervous system. A report of three cases. Bull Johns Hopkins Hosp 1943, 73: 405-415.

2. Parfrey, N.A. Improved diagnosis and prognosis of mucormycosis: A clinicopathological study of 33 cases. Medicine 1986, 65: 113-123.

3 Meyer, R.D., Rosen, P., Armstrong, D. Phycomycosis complicating leukemia and lymphoma. Ann Int Med 1972, 77: 871-879.

4. Benbow, E.W. \& Stoddart, R.W. Systemic zygomycosis. Postgrad Med J 1986, 62: 985-996.

5. Record, N.S.Jr. \& Ginder, D.R. Pulmonary phycomycosis without obvious predisposing factors. JAMA 1976, 235: 1256-1257. indicate the presence of this ubiquitous saprophyte, and not necessarily tissue invasion. Culture is required, however, to identify the fungal species. ${ }^{2}$ Recognition of the disease in life requires a high index of suspicion, and timely therapy with amphotericin B may aid in the survival of these patients with otherwise fatal disease. ${ }^{3}$

6. Baker, R.D. Pulmonary mucormycosis. Am J Pathol 1956, 32: 287-313.

7. Prout, G.R. Jr. \& Goddard, A.R. Renal mucormycosis: survival after nephrectomy and amphotericin B therapy. $N$ Engl J Med 1960, 263: 1246-1248.

8. Flood, H.D., O'Brien, A.M. \& Kelly, B.G. Isolated renal mucormycosis. Postgrad Med J 1980, 61: 175-176.

9. Calle, S. \& Klatsky, S. Intestinal phycomycosis (mucormycosis). Am J Clin Pathol 1966, 45: 264-272.

10. Deal, W.B. \& Johnson, J.E. Gastric phycomycosis: report of a case and a review of the literature. Gastroenterology 1969, 57: 579-586. 